\title{
Revista Brasileira de Enfermagem REBEn \\ Utilização de instrumento de registro de dados da saúde da criança e família e a prática do enfermeiro em atenção básica à saúde
}

\author{
Use of a tool for record of child and family health information \\ and nurse's practice in basic health care \\ Utilización del instrumento de registro de datos sobre la salud \\ del niño y de la familia y la práctica del enfermero en la atención básica a la salud
}

Juliana Coelho Pina

Enfermeira. Pós-Graduanda, nível Mestrado, da Escola de Enfermagem de Ribeirão Preto da Universidade de São Paulo (EERP/USP).

Débora Faleiros de Mello

Enfermeira. Professora Doutora do Departamento de Enfermagem Materno-Infantil e Saúde Pública da EERP/USP.

Simone Renata Lunardelo

Enfermeira. Mestre em Enfermagem em Saúde Pública. Núcleo de Saúde da Família IV - CSE/FMRP/USP.
Submissão: 08/06/2005

Aprovação: 04/03/2006

\section{RESUMO}

0 processo de crescimento e desenvolvimento infantil sofre influência de fatores diversos, sendo a família considerada como seu contexto primordial. Este estudo busca descrever a elaboração e utilização de um instrumento de observação, entrevistas e registro de dados sobre a criança e a família, na prática de enfermagem em atenção básica à saúde. Participaram desse estudo descritivo 10 famílias com crianças entre 0 e 24 meses de idade. As fontes de coleta de dados foram consulta aos prontuários, entrevistas e observação. A partir de uma revisão bibliográfica anterior, foi construído e aplicado um instrumento, evidenciando potencialidades para a sistematização da assistência de enfermagem e para a organização de informações e de melhores intervenções em saúde.

Descritores:Enfermagem pediátrica; Coleta de dados; Enfermagem.

\section{ABSTRACT}

Children's growth and development process is influenced by different factors and the family is considered as its primary context. This descriptive study aims at describing the elaboration and use of a tool in basic health care, aimed at observing, interviewing and registering data of children and families in nursing practice. Study participants were 10 families with children between 0 and 24 months of age. Data were collected by consulting patient records, interviews and observation. Based on a previous bibliographic review, an instrument was constructed and applied, which revealed possibilities for the systematization of nursing care, information organization and better health interventions.

Descriptors: Pediatric nursing; Data collection; Nursing.

\section{RESUMEN}

El proceso de crecimiento y desarrollo infantil sufre influencia de diversos factores y la familia es considerada como su contexto primordial. La finalidad de este estudio es describir la elaboración y utilización de un instrumento de observación, entrevistas y registro de datos sobre el niño y la familia, en la práctica de enfermería en la atención básica a la salud. Participaron de ese estudio descriptivo 10 familias con niños entre 0 y 24 meses de edad. Las fuentes de recopilación de datos fueron la consulta a los archivos, entrevistas y observación. A partir de una revisión bibliográfica anterior, fue construido y aplicado un instrumento, evidenciando potencialidades para la sistematización de la atención de enfermería y para la organización de informaciones y de mejores intervenciones en salud.

Descriptores: Enfermería pediátrica; Recolección de datos; Enfermería.

Pina JC, Mello DF, Lunardelo SR. Utilização de instrumento de registro de dados da saúde da criança e família e a prática do enfermeiro em atenção básica à saúde. Rev Bras Enferm 2006 maio-jun; 59(3): 270-3.

\section{INTRODUÇÃO}

O impacto de fatores biológicos, psicossociais (individuais e familiares) e ambientais no desenvolvimento infantil tem sido objeto de vários estudos, ampliando a idéia de contexto do desenvolvimento humano ${ }^{(1)}$. Outro fato que contribuiu para essa ampliação é a evolução do estudo das interações e relações no contexto familiar que, inicialmente, se focalizava na díade mãe-criança e, posteriormente, na tríade mãe-pai-criança, sendo que, a partir do final dos anos 80 , iniciou-se a preocupação com os sistemas familiares ${ }^{(2)}$.

É de suma importância considerar as modificações na dinâmica familiar e o redimensionamento do espaço privado que o nascimento de uma criança traz consigo, alterando papéis, modificando antigas relações e delineando novas funções ${ }^{(3)}$.

Para que uma criança cresça de maneira saudável, o contexto no qual ela se insere deve 
promover condições de proteção, atenção à saúde, socialização e educação ${ }^{(4)}$, papéis que são desempenhados pela família, pelos setores de educação e saúde e pela comunidade.

Na literatura, os temas saúde, doença, famílias e enfermeiras foram estudados separadamente durante muito tempo. A reciprocidade e a relação entre esses elementos são relativamente novos e, a partir desse entendimento, é possível promover um cuidado de saúde individual e familiar(5). Com essa ampliação dos cuidados de enfermagem junto às famílias torna-se necessário, em saúde da criança, estruturar a avaliação que envolve a criança, o grupo familiar e o ambiente, buscando abordar 0 desenvolvimento infantil.

Este estudo visa descrever a elaboração e utilização de um instrumento de entrevista e observação da criança e da família em visitas domiciliares e consultas de enfermagem, com vistas a contribuir para a prática do enfermeiro em atenção básica à saúde.

\section{METODOLOGIA}

Esta investigação configura um estudo descritivo sobre a elaboração e a utilização de um instrumento de registro de dados da saúde da criança e família para a prática do enfermeiro em visitas domiciliares e consultas de enfermagem. A pesquisa descritiva objetiva a observação, descrição e documentação de aspectos de uma situação, sendo delineada para caracterizar um fenômeno(6).

A partir da revisão de literatura, buscando reconhecer os instrumentos de coleta, registro e avaliação de dados acerca de crianças, observando seus conteúdos, formas de apresentação, aplicação e tipos de dados que fornecem, elaboramos o conteúdo e a forma do instrumento que propomos neste estudo. Após a revisão de literatura e elaboração do instrumento proposto, passamos à etapa da utilização do mesmo em uma realidade contextualizada, com aprovação em Comitê de Ética em Pesquisa.

O local selecionado para o estudo foi o Núcleo de Saúde da Família IV (NSF-IV), vinculado ao Centro de Saúde Escola (CSE-FMRP/USP), na cidade de Ribeirão Preto - SP, o qual foi escolhido por apresentar uma expressiva demanda de crianças.

Os participantes do estudo foram crianças de 0 a 24 meses de idade e suas famílias, cadastradas e acompanhadas no NSF-IV. As crianças foram selecionadas segundo mês de nascimento, a contar de 01 de abril de 2002 até 31 de março de 2004. Os responsáveis pelas crianças foram esclarecidos a respeito do caráter do estudo e seu objetivo, solicitando-se sua participação e consentimento, garantindo o sigilo dos dados coletados. Receberam também informações a respeito de sua liberdade em se recusar a participar ou retirar seu consentimento em qualquer fase da pesquisa, sem penalização alguma. Tendo ciência do exposto acima, aqueles que aceitaram participar assinaram o termo de consentimento livre e esclarecido.

Após consentimento, participaram dez famílias, sendo duas crianças para cada uma das seguintes faixas etárias: 0 a 3 meses, 4 a 8 meses, 9 a 12 meses, 13 a 18 meses e 19 a 24 meses.

As informações para compor o instrumento proposto foram coletadas em prontuários dos sujeitos observados e entrevistados e durante consultas e visitas domiciliares, na prática de enfermagem em saúde da família. Sua aplicação deu-se por meio de entrevista com os membros da família e observação do ambiente, da criança e das relações intrafamiliares.

Neste estudo, a coleta de dados em prontuários e por meio de entrevistas e observações, originou um volume de informações descritivas e narrativas. A análise dos dados deu-se a partir dessas informações, com vistas a descrever aspectos contemplados ou não nos tópicos do instrumento proposto, segundo sua pertinência e relevância para a assistência do enfermeiro na atenção básica à saúde.
Cabe ressaltar que não foram avaliadas e descritas as informações individuais das crianças e famílias, apenas realizamos uma breve caracterização geral da população estudada, demonstrando como as informações obtidas contribuem para o conhecimento da estrutura e dinâmica das famílias por parte da equipe. A confecção de um instrumento e sua utilização é o foco deste estudo, visando uma aproximação da realidade das famílias e ampliação da prática do enfermeiro.

\section{RESULTADOS E DISCUSSÃO}

A partir de uma revisão bibliográfica acerca do desenvolvimento infantil no contexto familiar, realizada anteriormente, as temáticas abordadas com maior freqüência nas produções foram aquelas relativas à família, ao contexto físico e social do desenvolvimento - englobando ambiente, interação, apego, comunicação e cultura - entrelaçando-se ao papel do enfermeiro no desenvolvimento infantil.

Ainda como fruto dessa revisão, identificamos a utilização de instrumentos no estudo do desenvolvimento infantil. Tais instrumentos são roteiros para observação, anamnese e entrevista, escalas, questionários e ainda um instrumento para apresentar os resultados da avaliação do crescimento e desenvolvimento. Eles têm a finalidade de analisar aspectos relativos à criança e às atividades que ela realiza, à relação mãe-filho, à estrutura e dinâmica familiares, ao ambiente e aspectos sócio-culturais. Alguns estudos utilizam vários instrumentos para alcançar seus propósitos, especialmente quando buscam realizar uma análise global do desenvolvimento infantil.

Esses aspectos levantados pela revisão bibliográfica nos apontam para a necessidade da construção de instrumentos que se destinem não apenas à avaliação do desenvolvimento infantil, mas à abordagem desse complexo processo, considerando todos os aspectos envolvidos - biológicos, sociais, culturais e interacionais.

Entre os 29 instrumentos levantados por revisão bibliográfica, 13 $(44,8 \%)$ foram localizados na Biblioteca Central do Campus de Ribeirão Preto da Universidade de São Paulo e em outras bibliotecas brasileiras através do Programa de Comutação Bibliográfica (Comut), sendo adquiridos. São eles: Denver Developmental Screening Test (DDST) Home Observation Measure of the Environment (HOME) ${ }^{(8,9)}$; Ficha de Acompanhamento do Desenvolvimento do Ministério da Saúde ${ }^{(10)}$; Procedimentos para Observação dos Organizadores da Psique de Spitz ${ }^{(10)}$; Roteiro para Observação do Ambiente Familiar ${ }^{(10)}$; Anamnese da Criança ${ }^{(10)} ;$ Anamnese da Família(10); Roteiro de Entrevistas com as Mães quanto ao Sentido atribuído ao Desenvolvimento Infantil( ${ }^{(10)}$; Roteiro de Observação da Relação Mãe/Criança(10); Anamnese de Acompanhamento ${ }^{(10)}$; Instrumento GAPEFAM (Grupo de Assistência, Pesquisa e Educação na Área da Saúde da Família) para Apresentação de Resultados da Avaliação do Crescimento e Desenvolvimento da Criança e suas Interações com a Família, Escola e Comunidade ${ }^{(11)}$; Inventario de Hechos y Eventos Vitales de la Vida Familiar (FILE) ${ }^{(12)}$; Guia Washington para promover o desenvolvimento de crianças pequenas ${ }^{(13)}$. Deve-se ressaltar que o Denver Developmental Screening Test (DDST) foi substituído pela sua nova edição, o Denver I/(14), que foi adquirido para esse estudo em detrimento do DDST.

Com base nesses instrumentos e também na experiência do Grupo Hospitalar Conceição - Porto Alegre, que utiliza um instrumento relevante para o seguimento de crianças, foram selecionados aspectos, agrupando-os nos seguintes tópicos: Histórico da Criança; Desenvolvimento Psicomotor; Histórico da Família; Apego e Interação Mãe-Filho-Família; Ambiente Físico e Social.

No tocante ao trabalho do enfermeiro em atenção primária à saúde, no Brasil, as ações e/ou intervenções de enfermagem são pouco estruturadas em relação a ter instrumentos para guiar a assistência. É preciso que haja a compreensão de que uma linguagem padronizada sobre a 
prática de enfermagem faz-se necessária, mas não se configura como um gesso. Ao contrário, visa colocar o fazer do profissional sob observação de forma ordenada, favorecendo, assim, seu refinamento, desenvolvimento e transformação constantes ${ }^{(15)}$. Nesse sentido, associações de classe, conselhos profissionais, instituições de ensino e órgãos governamentais têm preconizado e incentivado a sistematização da assistência de enfermagem (SAE). Tal sistematização contribui para a organização dos serviços de saúde, planejamento de ações e estabelecimento de prioridades, repercutindo em melhoria da qualidade da atenção à saúde da população.

Considerando-se que o presente estudo explora um instrumento em potencial para viabilizar a sistematização da assistência de enfermagem em saúde da criança, na estratégia saúde da família, espera-se que o produto dessa pesquisa seja utilizável na prática. Para tanto, faz-se necessário considerar as expectativas e necessidades do serviço de saúde em relação a esse instrumento.

As expectativas que nos foram manifestadas pelos profissionais de saúde do NSF-IV relacionavam-se com a estruturação do seguimento da criança, ou seja, a sistematização das consultas de puericultura, entrelaçada ao acompanhamento da família.

Além disso, após a aplicação do instrumento inicialmente proposto a duas crianças/famílias, notou-se a sua característica relativamente focada no tempo, pois sua estrutura não permitia um real seguimento dos sujeitos em estudo, à medida que não havia espaço para o registro de mudanças ocorridas (excetuando-se o tópico Desenvolvimento Psicomotor). Fazia-se necessário, sim, um registro histórico da criança e da família, porém aliado a uma flexibilidade à dinâmica e evolução características da criança e do grupo familiar.

Diante desse quadro, optou-se por realizar modificações no conteúdo e na forma do instrumento inicialmente proposto, visando atender à demanda dos profissionais de saúde daquela unidade e à necessidade de adequação para o seguimento de crianças e suas famílias, detectada pelas pesquisadoras. Essas modificações dizem respeito a:

- Agrupamento dos tópicos Histórico da Criança, Histórico da Família, Apego e Interação Mãe-Filho-Família e Ambiente Físico e Social nas primeiras páginas, de modo a facilitar o acesso a essas informações para direcionar a intervenção;

- Confecção de tabelas para o histórico da alimentação e vacinação da criança e para a estrutura familiar, organizando as informações e propiciando melhor acesso visual;

- Inclusão de um espaço de anotações, para serem registradas mudanças na estrutura/dinâmica familiares;

- Valorização do Acompanhamento, passando de subtópico do Histórico da Criança a um grande tópico, com uma folha para cada época da puericultura ( $1^{\text {a }}$ semana de vida - visita domiciliar - , 15 dias, $1^{\circ}$ mês, $2^{\circ}$ mês, $4^{\circ}$ mês, $6^{\circ}$ mês, $9^{\circ}$ mês, $12^{\circ}$ mês, $18^{\circ}$ mês e 2 anos), compondo um roteiro para a realização das consultas de puericultura com anamnese, exame físico, orientações a serem dadas e elementos aos quais o profissional deve dirigir sua atenção. O Desenvolvimento Psicomotor deixa de ser um tópico e é incorporado dentro do Acompanhamento.

Feitas essas modificações, prosseguiu-se com a aplicação do instrumento final (APÊNDICE 1), que se demonstrou eficaz em reunir as informações desejadas.

Nesse ponto, pode-se questionar se 0 instrumento que propomos destina-se apenas à coleta de dados ou realmente consiste em um instrumento de abordagem mais ampla do desenvolvimento infantil no contexto familiar. Quanto a isso, ressaltamos que a obtenção de dados não se dá um em único momento, mas é um processo que requer vários encontros, dentro da dinâmica do Programa de Saúde da Família (visitas domiciliares, consultas, acompanhamentos, etc.), pressupondo a criação de vínculo e conhecimento entre os profissionais e a família. Além disso, os próprios dados coletados nos traduzem o modo de vida de cada família, como ele poderia influenciar o desenvolvimento de cada criança e nos conduzem a uma reflexão de como agir.

Nesse sentido, o levantamento de dados que realizamos nos indicam que, em geral, a população do estudo mora em microárea de risco (favela), com condições de saneamento básico precárias, sem espaço potencial para as crianças nos cômodos e recebem ajuda do governo. Os pais possuem baixa escolaridade ( $1^{\circ}$ grau incompleto), não são ligados por laços oficiais, sendo comum a mãe ter filhos de pais diferentes e ser solteira. Na divisão das responsabilidades, prevalece o cuidado da casa e dos filhos a cargo da mulher e o sustento da família a cargo do homem, embora a administração do dinheiro seja compartilhada por ambos. Outra particularidade é a tendência dos filhos mais velhos (porém ainda crianças) serem responsáveis pelo cuidado dos mais novos, enquanto os pais trabalham, pois a creche do bairro não absorve toda a demanda.

Apesar de se tratar de uma população pobre, na qual o aleitamento materno traria benefícios não somente à saúde das crianças mas também financeiros, pela economia de alimentos, o que se observa é a introdução precoce de outros líquidos e alimentos, especialmente quando a avó mora com a família e tende a assumir o lugar da mãe.

Mesmo em meio a situações socioeconômicas precárias, a maioria das crianças estudadas encontra-se dentro do esperado para o desenvolvimento neuropsicomotor, sugerindo que podem haver fatores protetores e de enfrentamento eficazes no grupo familiar e na comunidade.

\section{CONSIDERAÇÕES FINAIS}

Com base nesse estudo descritivo, julgamos que o instrumento final tem o potencial de propiciar uma base segura para o seguimento da criança e sua família, pois nele o enfermeiro encontra, de forma ordenada, informações sobre antecedentes pré-natais, parto, histórico alimentar e vacinal, estrutura e dinâmica familiares, ambiente físico, relações intrafamiliares e sociais e acompanhamento da criança até 0 $2^{\circ}$ ano de vida. Acreditamos que, desse modo, o profissional capacitado poderá interligar as informações disponíveis, guiando a assistência prestada a nível individual e familiar.

Consideramos, no entanto, que a viabilidade dessa situação depende de análises mais profundas sobre o produto final desse estudo, aplicando-o em amostras maiores, em um seguimento contínuo desde o nascimento, a fim de verificar sua capacidade de armazenar e disponibilizar as informações necessárias ao acompanhamento de enfermagem às crianças menores de dois anos e suas famílias, na atenção básica à saúde.

\section{REFERÊNCIAS}

1. Halpern R, Giugliani ERJ, Victoria CG, Barros FC, Horta BL. Fatores de risco para suspeita de atraso no desenvolvimento neuropsicomotor aos doze meses de vida. J Ped 2000; 76(6): 421-8.

2. Dessen MAC. Interações e relações no contexto familiar: questões teóricas e metodológicas. Psicol Teoria Pesq 1994; 10(2): 213-20.

3. Martins DC. $O$ seguimento de enfermagem às crianças menores de um ano de idade em uma unidade de saúde da família de Ribeirão Preto, SP [dissertação]. Ribeirão Preto (SP): Escola de Enfermagem de Ribeirão Preto, USP; 2003.

4. Myers RG. Los doce que sobreviven: fortalecimiento de los programas de desarrollo para la primera infancia en el Tercer Mundo. Washington (DC): Organización Panamericana de la Salud; 1993. 
5. Wright LM, Leahey M. Enfermeiras e famílias: um guia para a avaliação e intervenção na família. São Paulo (SP): Roca; 2002.

6. Polit DF, Hungler BP. Fundamentos de pesquisa em enfermagem. $3^{\text {a }}$ ed. Porto Alegre (RS): Artes Médicas; 1995.

7. Moraes MCL, Moron AF. Alguns pressupostos teóricos para "um emprego" do conceito de desenvolvimento. Rev Bras Cresc Des Hum 1999 jan-jun; 9(1): 35-40.

8. Black MM, Dubowitz H, Hutcheson J, Howard JB, Starr RH. A randomized clinical trial of home intervention for children with failure to thrive. Pediatrics 1995 jun; 95(6): 807-14.

9. Bastos ACS, Almeida-Filho N. Variables económicosociales, ambiente familiar y salud mental infantil en un área urbana de Salvador (Bahia), Brasil. Acta Psiq Psicol Amer Lat 1990; 36(3/4): 147-54.

10. Siqueira AAF, Oliveira DC, Rabinovich EP, Santos NG. Instrumentos para o acompanhamento e avaliação do desenvolvimento infantil na Atenção Primária à Saúde. Rev Bras Cresc Des Hum 1992 jul-dez;
2(2): 59-99.

11. Rosa MC, Pelissari DP, Rinaldi GS. Boletim "Processo de viver com cidadania". Texto \& Contexto Enfermagem 1995 jan-jun; 4(1): 127-38.

12. Castro MSM. Eventos estresores que influyen en la familia para el abandono parcial del menor de cuatro años. Actual Enfermería 1999 jun; 2(2): 9-20.

13. Veríssimo MDLOR, Sigaud CHS. O acompanhamento do crescimento e desenvolvimento da criança. In: Sigaud CHS, Veríssimo MDLOR (organizadoras). Enfermagem pediátrica: o cuidado de enfermagem à criança e ao adolescente. São Paulo (SP): EPU; 1996. p. 137-62.

14. Frankenburg WK, Doods J, Archer P, Shapiro H, Bresnick B. The Denver II: a major revision and restandardization of the Denver Development Screening Test. Pediatrics 1992; 89(1): 91-7.

15. Cruz DALM. Fenômenos e intervenções de enfermagem: desatando nós conceituais. In: Associação Brasileira de Enfermagem (ABEn). Sistemas de classificação da prática de enfermagem: um trabalho coletivo. João Pessoa (PB): Idéia Ed.; 2000. p. 28-36. 